\title{
Management of a Small Paracentral Corneal Perforation Using latrogenic Iris Incarceration and Tissue Adhesive
}

\author{
Akira Kobayashi Hideaki Yokogawa Kazuhisa Sugiyama \\ Department of Ophthalmology, Kanazawa University Graduate School of \\ Medical Science, Kanazawa, Japan
}

\section{Key Words}

Cyanoacrylate $\cdot$ Tissue adhesive $\cdot$ Corneal perforation · Iris incarceration

\begin{abstract}
Background: Surgical intervention for corneal perforation is indicated when the anterior chamber does not reform within a short period of time. Herein, we report the successful management of a small paracentral corneal perforation using autologous iris incarceration and tissue adhesive.

Case: A 41-year-old man developed a small paracentral corneal perforation ( $0.5 \mathrm{~mm}$ in size) in the right eye, while the treating physician attempted to remove the residual rust ring after removal of a piece of metallic foreign body.

Observations: The eye was initially managed with a bandage soft contact lens to ameliorate the aqueous leakage; however, without success. latrogenic iris incarceration of the wound was first induced, followed by application of cyanoacrylate tissue adhesive to the perforated site. As a result, the anterior chamber was immediately reformed and maintained. Complete corneal epithelialization of the perforation was achieved in 2 months without visual compromises.

Conclusions: Cyanoacrylate tissue adhesive with iatrogenic incarceration of the autologous iris was effective in treating this type of small corneal perforation. This technique is simple and potentially useful for small paracentral corneal perforations outside the visual axis and without good apposition.
\end{abstract}




\section{Introduction}

Corneal perforation caused by small corneal punctures can sometimes be managed with a bandage soft contact lens [1], patching, and/or tissue adhesive [2-4]. However, surgical intervention is usually indicated when the anterior chamber does not reform within a short period of time. Herein, we report the successful experience of managing a small paracentral corneal perforation without good apposition, which could not be tamponaded by a soft bandage lens only, with iatrogenic iris incarceration followed by cyanoacrylate tissue adhesive.

\section{Case Report}

A 41-year-old man noted irritation in the right eye and was diagnosed to have a piece of metallic foreign body in his right cornea. The foreign body was first removed with a needle without difficulty. Iatrogenic corneal perforation was encountered, while the attending physician attempted to remove the residual rust ring with a corneal burr. The patient was immediately referred to our department for further management. Visual acuity in his right eye decreased to 20/200 upon presentation. Slitlamp examination revealed a paracentral corneal penetration with a diameter of approximately 0.5 $\mathrm{mm}$ (fig. 1). The anterior chamber was flat, and active aqueous leakage was confirmed by a positive Seidel test with fluorescein. Orbital computed tomography and X-ray film showed no intraocular foreign body. The left eye was completely unremarkable with an uncorrected visual acuity of 20/15. The patient was admitted and managed with a soft bandage contact lens. Intravenous antibiotics with cefozopran hydrochloride were initiated, in conjunction with prophylactic topical antibiotic with $0.5 \%$ levofloxacin three times daily. Microbiological culture of the conjunctival sac was performed, which yielded negative growth. After $48 \mathrm{~h}$ of treatment, there was no significant improvement in the right eye. Concerns were raised about possible complications such as secondary angle closure glaucoma and prolonged hypotony. Since the puncture was located paracentrally near the visual axis, simple primary closure of the wound or corneal patch graft was deemed undesirable. Therefore, application of a cyanoacrylate adhesive to restore the cornea tectonically was preferred.

The cornea was anesthetized with $2 \%$ lidocaine hydrochloride. As the wound was completely 'punched out', injected viscoelastic materials in the anterior chamber came out from the wound, which made it quite difficult to dry the surface of the cornea for application of tissue adhesive. Therefore, to provide a necessary support for the gluing, amniotic membrane plugging was tried, but it was too slippery to be fixed on the wound. Finally, the autologous iris was used for this case. Viscoelastic material was injected through the punctured wound to reform the anterior chamber. A paracentesis port was created. A blunt 27-gauge needle (with a 2-mm bent tip) attached to a viscoelastic injecter was gently inserted into the anterior chamber through the paracentesis. Using the bent tip, a small tug of iris was incarcerated into the wound from the posterior wound surface. Care was taken not to touch or injure the corneal endothelium or anterior capsule of the lens. Meticulous drying of the wound and incarcerated iris was performed and a tiny amount of ethyl-2-cyanoacrylate adhesive (Aron Alpha A; Sankyo Co. Ltd., Tokyo, Japan) was applied to the wound to seal it completely. Attention was paid to ensure that the glue did not spread on to the surrounding cornea. A bandage soft contact lens was then placed over the cornea. The corneal puncture was thus successfully closed without any sutures, and the anterior chamber remained well formed. Postoperatively, the patient was treated with topical $0.1 \%$ fluorometholone and $0.5 \%$ levofloxacin three times daily. The adhesive remained on the cornea for 2 months with negative Seidel tests. The glue eventually detached and the cornea was completely epithelialized. Postoperatively, the corneal surface was smooth showing a small residual scar (fig. 2). Uncorrected final visual acuity improved to 20/20. Pupillary distortion was observed when the iris was dilated; however, the patient felt no visual disturbance. 


\section{Discussion}

Herein, we reported a case of iatrogenic paracentral corneal perforation without good apposition successfully treated with autologous iris incarceration and cyanoacrylate adhesive. Conventionally, small corneal perforations were treated with stitches with or without corneal patch graft, tissue adhesives [2-4], and recently with amniotic membrane and fibrin glue [5]. Corneal suturing may result in high astigmatism. According to Leibowitz [1], the indications for the use of a bandage soft contact lens to treat a corneal laceration include small wounds with good apposition, good alignment of the wound edges, and no incarceration or prolapse of the uvea or lens. Corneal puncture wounds of less than $2.0 \mathrm{~mm}$ in size can usually heal without being sutured [2]. In this case, the wound was relatively small (less than $1 \mathrm{~mm}$ ), but the apposition of the wound was not optimal due to a sharply demarcated punch, which made restoring the anterior chamber extremely difficult using a bandage soft contact lens only. In cases with good apposition, a small amount of viscoelastic materials through the perforation site is often enough to allow safe application of tissue adhesive without gluing intraocular contents.

The technique described in this case appears useful secondary to the minimal residual scarring and related astigmatism. A second advantage of this technique is that the iris is introduced to the perforation site from inside the eye in a controlled manner to prevent excessive iris prolapse and provide a stable support for the tissue adhesive. This is more adequate than the use of an amniotic membrane, which is very slippery and difficult to be fixed on the wound. Furthermore, this technique requires no foreign tissue, thereby eliminating the risk of immunological tissue reactions. The tissue adhesive may also theoretically circumvent the potential risks of epithelial downgrowth by preventing corneal epithelium from growing directly over the iris. This technique is safe and effective for paracentrally located corneal perforation; however, significant synechiae involving the angle could prove to be problematic when the perforation is more peripheral. Very small Descemet's membrane transplantation instead of iris incarceration may be the other option for this situation.

In conclusion, application of cyanoacrylate adhesive following iatrogenic incarceration of the iris seems to be simple and effective in managing small paracentral corneal perforations without good apposition.

\section{Disclosure Statement}

The authors declare that they have no conflict of interest. 


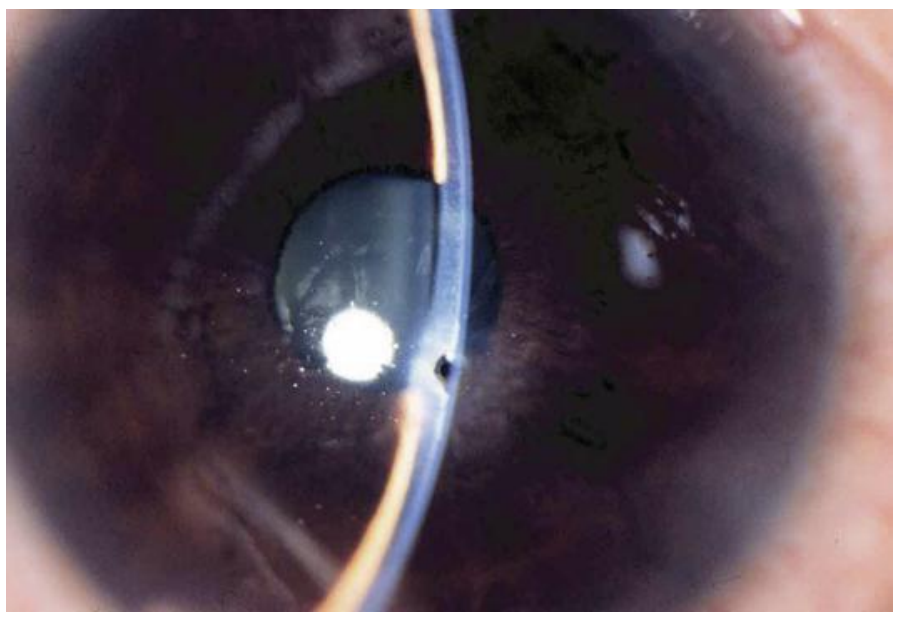

Fig. 1. Anterior photograph of the right eye at the initial visit. The cornea has been completely punched through by the drill and the anterior chamber is absent.

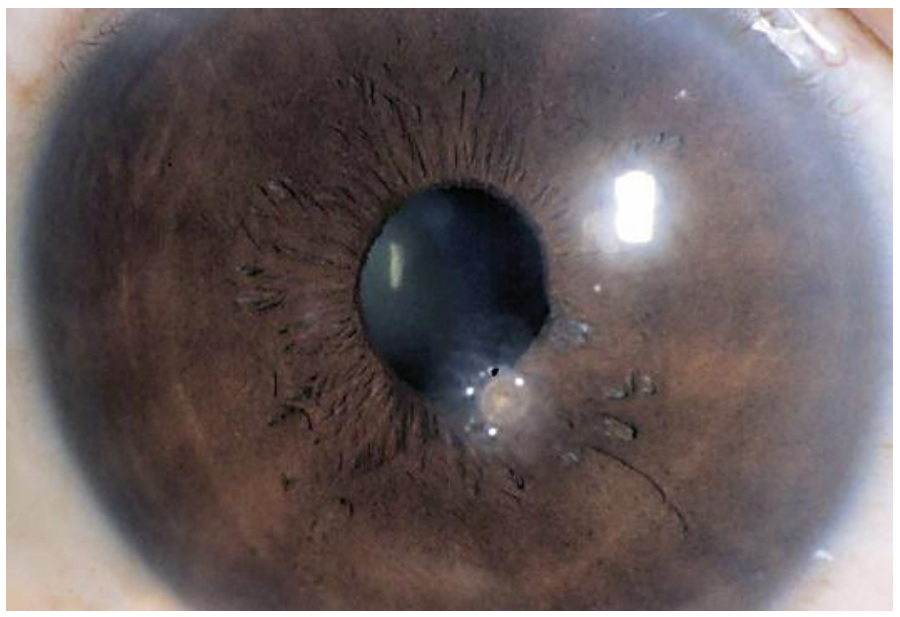

Fig. 2. Postoperative stage of the wound. Two weeks postoperatively, the corneal surface is smooth and shows a small scar. The anterior chamber is deep, with the edge of the pupil (5 o'clock position) attached behind the posterior cornea.

\section{References}

1 Leibowitz HM: Hydrophilic contact lenses in corneal disease. IV. Penetrating corneal wounds. Arch Ophthalmol 1972;88:602.

2 Parrish CM, Chandler JW: Corneal trauma; in Kaufman HE, Barron BA, McDonald MB (eds): Cornea Second edition. Washington: Butterworth-Heinemann, 1997, pp 633-672.

3 Webster RG, Slansky HH, Refojo MF, et al: The use of adhesive for closure of corneal perforations. Arch Ophthalmol 1968;80:705-709.

-4 Taravella MJ, Chang CD: 2-Octyl cyanoacrylate medical adhesive in treatment of a corneal perforation. Cornea 2001;20:220-221.

5 Duchesne B, Tahi H, Galand A: Use of human fibrin glue and amniotic membrane transplant in corneal perforation. Cornea 2001;20:230-232. 